\title{
Pachyonychia congenita Typ III
}

\author{
Pachyonychia Congenita Type III
}

Autoren

Institut

\section{F. Oberthür, A. Wagner, C. Bayerl}

Klinik für Dermatologie und Allergologie, Städtisches Klinikum Wiesbaden, HSK, Wilhelm-Fresenius-Klinik GmbH Lehrkrankenhaus der Universität Mainz

\section{Bibliografie}

DOI $10.1055 / \mathrm{s}-2008-1077551$

Akt Dermatol 2008; 34 :

336-339 @ Georg Thieme

Verlag KG Stuttgart · New York ISSN 0340-2541

Korrespondenzadresse

\section{Frank Oberthür}

Klinik für Dermatologie

und Allergologie

Städtisches Klinikum

Wiesbaden

HSK, Wilhelm-Fresenius-Klinik $\mathrm{GmbH}$

Aukammallee 39

65191 Wiesbaden

E-mail: Frank.Ob@gmx.de

\section{Zusammenfassung \\ $\nabla$}

Die Pachyonychia congenita (PC) ist eine meist autosomal dominante Erbkrankheit mit ektodermalen Dysplasien, bei der 4 unterschiedliche Typen beschrieben sind. Wir berichten über eine 76-jährige Patientin mit PC Typ III. Seit der Geburt litt sie rezidivierend unter prallen schmerz-

\section{Einleitung}

Die Pachyonychia congenita (PC) ist eine seltene, meist autosomal dominant vererbte, ektodermale Dysplasie mit variablem Phänotyp $[4,7,15]$. Es existieren mehrere Einteilungen der PC. Feinstein et al. [8] unterscheiden nach dem klinischen Bild vier verschiedene Typen. Beim Typ III zeigen sich neben der pathognomonischen Pachyonychie, den palmoplantaren Hyperkeratosen und den follikulären Keratosen, multiple epidermale Zysten, palmoplantare Blasenbildung, Hyperhidrose, neonatale Zähne, Cheilitis angularis, Korneaveränderungen sowie Katarakt [23,24].

\section{Krankengeschichte}

Die 76-jährige Patientin berichtete, seit der Geburt unter Wunden an den Füßen zu leiden. Insbesondere kam es an den Fußsohlen zur Ausbildung von prallen schmerzhaften Blasen, welche sich rezidivierend seit dem Kleinkindesalter noch vor Erlernen des Laufens entwickelten. Ferner leide sie ebenfalls seit der Geburt unter verdickten Finger- und Zehennägeln. Bisher kam es im Sommer regelmäßig zu einer Verschlechterung und im Winter zu einer Verbesserung des Hautbefundes. Abgeheilt seien die Hautveränderungen nicht. Vor vier Jahren kam es letztmalig zu einer starken Exazerbation der Hautveränderungen mit großen „offenen Stellen“ an den Fü- haften Blasen an den Füßen. Ferner bestanden plantare Hyperkeratosen, multiple epidermale Zysten, Cheilitis angularis, orale Leukoplakie, Katarakt sowie die typische pyramidenartige Verdickung der Fingernägel. Pathogenese, Diagnostik sowie die therapeutischen Möglichkeiten bei PC werden vorgestellt.

ßen. Seitdem war sie bis 3 Wochen vor stationärer Aufnahme weitgehend erscheinungsfrei. Ferner bestand bei der Patientin eine Katarakt. Eine Intelligenzminderung war nicht erkennbar. Die inzwischen verstorbene Tochter hatte unter den gleichen Symptomen gelitten, die übrige Familienanamnese war unauffällig.

\section{Lokalbefund \\ $\nabla$}

An der linken lateralen Fußkante und am linken Außenknöchel fand sich je eine zirka handtellergroße Hyperkeratose, z. T. mit schlaffer blasiger Abhebung ( $\bullet$ Abb. 1). Die Umgebung war gerötet. Am rechten Fuß zeigte sich lediglich an der lateralen Ferse eine zirka $5 \mathrm{~cm}$ große schlaffe Blase mit hyperkeratotischem Dach. Beidseits plantar und insbesondere unter den Metatarsalköpfchen bestanden ausgeprägte Hyperkeratosen. Die Finger- und Fußnägel waren gelblich verfärbt und pyramidenartig verdickt ( $\bullet$ Abb. 2). Im Gesicht und an beiden Oberarminnenseiten bestanden multiple, ca. stecknadelkopfgroße hautfarbene bis erythematöse Papeln ( $\bullet$ Abb.3). Enoral zeigte sich an den Wangen und auf der Zunge eine Leukoplakie. Die Zähne waren nicht verändert. Im Bereich der Mundwinkel bestanden kleinere Rhagaden. 


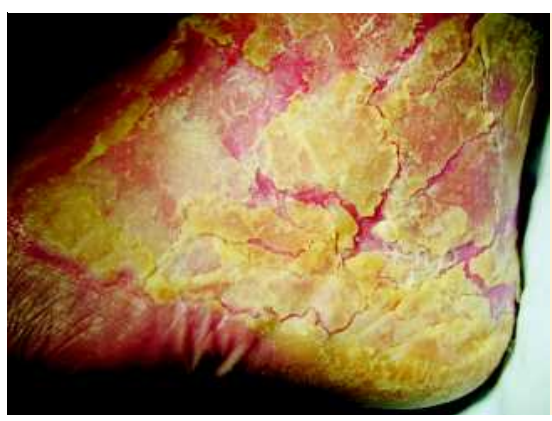

Abb. 1 Lateraler Fuß mit blasiger hyperkeratotischer Abhebung der Haut.

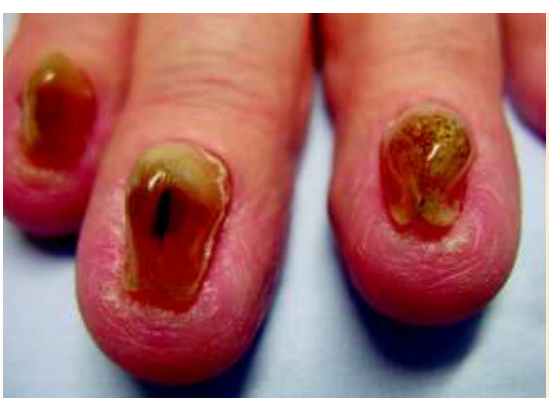

Abb. 2 Gelbliche Verfärbung und pyramidenartige Verdickung der Fingernägel.

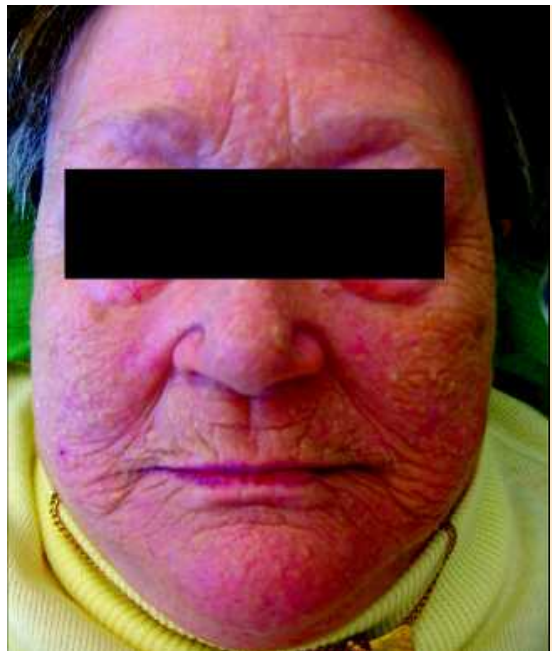

Abb. 3 Gesicht mit multiplen Steatozystomen.

\section{Laborbefunde}

7

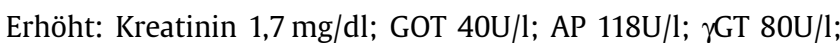
Triglyceride $210 \mathrm{mg} / \mathrm{dl}$; CRP 3,9 mg/dl; Leukozyten 14,3/nl; BSG 28/61 mm n. W.

Erniedrigt: HDL $42 \mathrm{mg} / \mathrm{dl}$.

Die übrigen Laborparameter der klinischen Chemie und des Differenzialblutbildes waren unauffällig.

\section{Weitere Untersuchungsbefunde}

$\nabla$

Dermato-Histologie: Am Blasenrand des lateralen Fußes zeigte sich in der Biopsie eine akanthotisch verbreiterte Epidermis mit orthokeratotischer Verhornung. Es fand sich eine schwammig aufgelockerte Spongiose sowie ein intrazelluläres Ödem im Bereich der Epidermis. In einem Randbereich zeigte sich eine intraepidermale Spaltbildung. Im Korium fand sich ein meist perivaskulär orientiertes entzündliches Infiltrat aus Histiozyten, Lymphozyten und Granulozyten und deutliche Erythrozytenex-

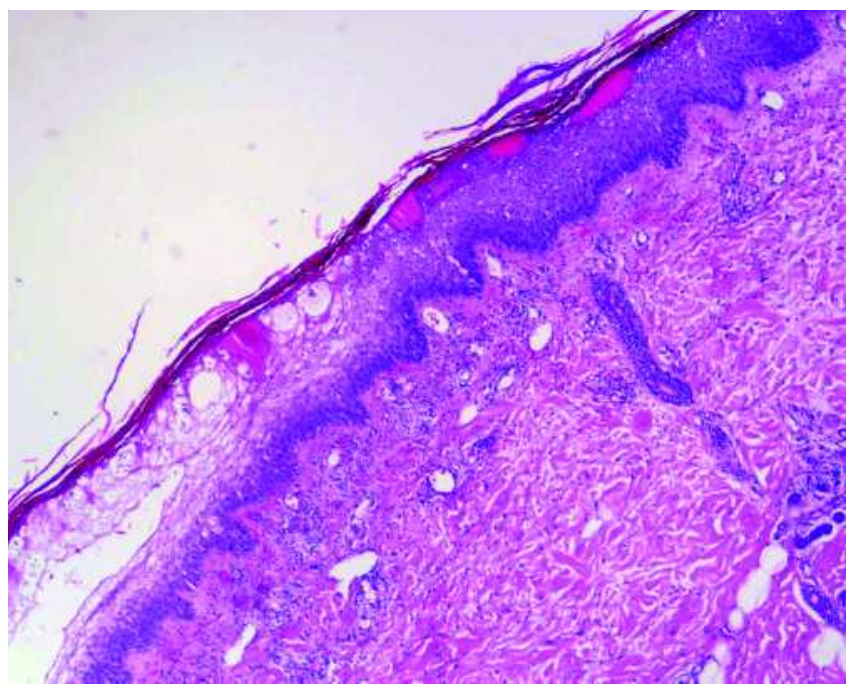

Abb. 4 Histologie aus dem Randbereich einer Blase.

travasate. Ödematöse Auflockerung des kollagenen Bindegewebes ( $\bullet$ Abb. 4).

Mikrobiologischer Befundbericht: Multiple Kolonien Enterococcus species, z. B. sensibel auf Ciprofloxacin.

Minor Schweißtest: Palmoplantar zeigte sich eine Anhidrose.

\section{Therapie und Verlauf \\ $\nabla$}

Lokal verwendeten wir 3\%ige Salizylvaseline und 10\%ige Harnstoff-Salbe sowie feuchte $\mathrm{NaCl}$-Umschläge. Mittels regelmäßiger vorsichtiger mechanischer Abtragung kam es zu einer Reduktion der Hyperkeratosen. Oben genannte Lokaltherapeutika brannten jedoch auf der Haut und wurden insgesamt schlecht vertragen. Die weitere Therapie erfolgte dann mit Framycetin-Salbe und Tazaroten-Gel im morgend-/abendlichem Wechsel.

Systemisch wurde eine Therapie mit Acitretin $10 \mathrm{mg} 1 \times$ tgl. eingeleitet. Aufgrund der bakteriellen Superinfektion mit Enterococcus species erfolgte ergänzend eine antibiotische Therapie mit Ciprofloxacin 500mg-Tbl. $2 \times$ tgl.

Unter o. g. Therapie kam es allmählich zu einer deutlichen Besserung des Lokalbefundes.

\section{Ätiopathogenese und Einteilung der PC}

$\nabla$

Diese autosomal-dominanten Verhornungsstörungen sind durch eine symmetrische Verdickung aller Nägel in Assoziation mit palmoplantaren und follikulären Hyperkeratosen sowie oralen Leukokeratosen gekennzeichnet $[4,7,15]$. Die PC wurde erstmals von Müller 1904 [20], Wilson 1905 [28] und dann 1906 von Jadassohn und Lewandowsky [13] beschrieben. Bisher wurden insgesamt ca. 200 Fälle publiziert.

So verwundert es nicht, dass aufgrund der verschiedenartigen Ausprägung der Erkrankung unterschiedliche Einteilungen existieren. Die aktuelle Einteilung nach Feinstein et al. [8] erfolgt in 4 verschiedene Typen: Der häufigste Typ I (56,2\%), das JadassohnLewandowsky-Syndrom, ist neben der bei allen Varianten vorkommenden Pachyonychie durch palmoplantare Hyperkeratosen, follikuläre Keratosen sowie orale Leukoplakie gekennzeichnet. Der seltenere Typ II (24,9\%), das Jackson-Lawler-Syndrom, 
Tab. 1 Pachyonychia congenita - Einteilung nach Feinstein et al.

\begin{tabular}{|c|c|c|c|c|}
\hline Klinische Symptome & $\begin{array}{l}\text { Typ I } \\
\text { Jadassohn-Lewan- } \\
\text { dowsky- Syndrom }\end{array}$ & $\begin{array}{l}\text { Typ II } \\
\text { Jackson-Lawler- } \\
\text { Syndrom }\end{array}$ & Typ III & Typ IV \\
\hline Hyperkeratose der Nägel & + & + & + & + \\
\hline Palmoplantare Hyperkeratosen & + & + & + & + \\
\hline Follikuläre Keratosen & + & + & + & + \\
\hline Orale Leukoplakie & + & - & \pm & \pm \\
\hline Palmare und plantare Blasenbildung & - & + & + & + \\
\hline Palmare und plantare Hyperhidrose & - & + & + & + \\
\hline Multiple Epidermoidzysten & - & + & \pm & \pm \\
\hline Assoziation mit Steatocystoma multiplex & - & + & $?$ & $?$ \\
\hline Nataler/pränataler Zahnstatus & - & \pm & + & + \\
\hline Cheilitis & - & - & + & + \\
\hline Korneale Dyskeratosen & - & - & + & + \\
\hline Katarakt & - & - & + & + \\
\hline Laryngeale Defekte & - & - & - & + \\
\hline Schwerhörigkeit & - & - & - & + \\
\hline Geistige Retardierung & - & - & - & + \\
\hline Haaranomalien & - & - & - & + \\
\hline
\end{tabular}

grenzt sich durch das zusätzliche Auftreten von palmoplantarer Blasenbildung, Hyperhidrose, multiplen Epidermoidzysten und neonatalem Zahnstatus bei Fehlen der Schleimhautveränderungen ab. Beim Typ III (11,7\%) bestehen außerdem eine Cheilitis angularis sowie Korneaveränderungen und Katarakt, daneben finden sich beim Typ IV (7,2\%) Leukokeratosen des Kehlkopfs (chronische Heiserkeit), Schwerhörigkeit, geistige Retardierung und Haaranomalien $[4,15,23,24]$.

Bei der Pachyonychia congenita tarda treten die typischen Nagelveränderungen erst in der 2. oder 3. Lebensdekade auf $[11,16]$.

\section{Therapie}

Die Therapieoptionen sind leider unbefriedigend, da keine kausale Therapie existiert. Die Nagelverdickungen können mit keratolytischen Externa (20\% - 40\% Harnstoff oder 15\% - 20\% Salizylsäure) schonend reduziert werden, alternativ empfiehlt sich auch eine mechanische Abtragung, allerdings sollte hier vorsichtig vorgegangen werden, um Verletzungen mit nachfolgender Infektion zu verhindern. Unsere Patientin erzielte mit letzterer Methode und anschließender Versiegelung mit Nagellack gute kosmetische Ergebnisse. Die Hyperkeratosen können zum Beispiel mit Acitretin behandelt werden, langfristig können jedoch nur partielle Besserungen erzielt werden. Vor allem die schmerzhafte Blasenbildung bleibt in der Regel unbeeinflusst, weswegen viele Patienten die Therapie im Verlauf wieder abbrechen. Follikuläre Keratosen können mit mäßigem Erfolg mit Alpha-Hydroxysäure oder keratolytischen Externa behandelt werden. Epidermoidzysten können aufgestochen und exprimiert werden. Bei Neigung zu Blasen an den Füßen sollte vor allem auf weiches, orthopädisches Schuhwerk geachtet werden. Bei einigen Patienten konnten auch gute Erfolge durch Behandlung der oft bestehenden Hyperhidrose durch Aluminiumsalze oder Botulinumtoxin erzielt werden [4,17].

Da die Patienten aufgrund des Leitsymptoms der Pachyonychie zunächst den Dermatologen aufsuchen, ist es wichtig, die seltene PC in der Differenzialdiagnose der Nageldystrophien nicht zu vergessen und ggf. eine gründliche interdisziplinäre Abklärung
(Innere Medizin, HNO, Ophthalmologie) zu veranlassen, um rechtzeitig auf evtl. zusätzlich bestehende Erkrankungen aufmerksam zu werden [15].

\section{Abstract}

\section{Pachyonychia Congenita Type III}

$\nabla$

Pachyonychia congenita (PC) is a rare ectodermal dysplasia with variable expression. The condition is usually inherited as an autosomal dominant trait. Feinstein et al. differentiate four clinical types of PC. We report a 76 year old woman with PC type III. Since birth, the patient showed recurrent plantar plump painful blisters, plantar hyperkeratosis, multiple epidermal cysts, cheilitis angularis, oral leukokeratosis, cataract as well as typical pyramid-like swelling of the fingernails. Pathogenesis, diagnostic criteria and treatment approaches are discussed.

\section{Literatur}

1 Benjamin B, Parsonsn DS, Molloy HF. Pachyonychia congenita with laryngeal involvement. Int J Pediatr Otorhinolaryngol 1987; 13: 205 209

2 Bensa AF, Dalac S, Beer F et al. Pachyonychia congénitale, neurofibromatose et polyneuropathie sensitivomotrice. Ann Dermatol Venereol 1995; $122: 428-431$

3 Besser FS, Moynahan EJ. Pachyonychia congenita with epidermal cysts and teeth at birth: 4th generation. Br J Dermatol 1971; 84: 95 - 96

4 Braun-Falco O, Plewig G et al. Dermatologie und Venerologie. Springer Medizin Verlag 2005; 5: 966-967

5 Chevrant-Breton J, Lezoraine C et al. Syndrome de Jackson-Lawler: étude de deux familles. Ann Dermatol Venereol 1991; 118: 781 - 784

6 Clementi M, Cardin deStefani E et al. Pachyonychia congenita JacksonLawler type: a distinct malformation syndrom. Br J Dermatol 1986; 114: $367-370$

7 Corden LD, McLean WH. Human keratin diseases: hereditary fragility of specific epithelial tissues. Exp Dermatol 1996; 5: 297-307

8 Feinstein A, Friedman J, Schewach-Millet M. Pachyonychia congenita. J Am Acad Dermatol 1988; 19: 705-711

9 Haber RM, Rose TH. Autosomal rezessive Pachyonychia congenita. Arch Dermatol 1986; 122: 919-923 
10 Hoting E, Wassilew SW. Systemische Retinoidtherapie mit Etretinat bei Pachyonychia congenita. Hautarzt 1985; 36: 526-528

11 Iraci S, Bianchi L, Gatti S et al. Pachyonychia congenita with late onset of nail dystrophy - a new clinical entity? Clin Exp Dermatol 1993; 18: $478-480$

12 Jackson ADM, Lawler SD. Pachyonychia congenita: a report of six cases in one family. With a note on linkage data. Ann Eugen 1950/51; 16: $141-146$

13 Jadassohn J, Lewandowsky F. Pachyonychia congenita. Keratosis disseminata circumscripta (folliculari). Tylomata. Leukokeratosis linguae, vol 1. In: Jacobs Ikonographia Dermatologica. Berlin: Urban \& Schwarzenberg, 1906: 29-31

14 Kumer L, Loos HO. Kongenitale Pachyonychie (Riehl Type). Wien Klin Wochenschr 1935; 48: 174-178

15 Löchner J, Mohr B et al. Pachyonychia congenita Typ II (Jackson-LawlerSyndrom). Hautarzt 2000; 51: $192-195$

16 Lucker GP, Steijlen PM. Pachyonychia congenita tarda. Clin Exp Dermatol 1995; 20: 226-229

17 Milstone LM, Fleckman P et al. Treatment of pachyonychia congenita. J Investig Dermatol Symp Proc 2005; 10: $18-20$

18 Moldenhauer E, Ernst K. Das Jadassohn-Lewandowsky-Syndrom. Hautarzt 1968; 19: 441 - 447

19 Moldenhauer E, Seidel $R$. Is sebocystomatosis a symptom of the Jadassohn-Lewandowsky syndrom? Dermatol Monatsschr 1973; 159: $540-543$
20 Müller C. Zur Kasuistik der kongenitalen Onychogryphosis. Münch Med Wochenschr 1904; 51: 2180-2182

21 Munro CS, Carter S, Bryce S et al. A gene for pachyonychia congenita is closely linked to the gene cluster on 17q12-q21. J Med Genet 1994; 31: $675-678$

22 Premalatha S, Thambiah AS. Pachyonychia congenita with cardiac involvement. Arch Dermatol 1977; 113: 687

23 Leachman SA, McLean WHI et al. Preface to Pachyonychia Congenita Symposium Proceedings. J Investig Dermatol Symp Proc 2005; 10: $1-2$

24 Leachman SA, Kaspar RL et al. Clinical and pathological features of pachyonychia congenita. J Investig Dermatol Symp Proc 2005; 10: 3-17

25 Smith FJ, Corden LD, Rugg EL et al. Missense mutations in keratin 17 cause either pachyonychia congenita type 2 or a phenotype resembling steatocystoma multiplex. J Invest Dermatol 1997; 108: 220-223

26 Todd P, Garioch J, Rademaker M et al. Pachyonychia congenita complicated by hidradenitis suppurativa: a family study. Br J Dermatol 1990; 123: $663-666$

27 Velasquez JP, Bustamante J. Sebocystomatiasis with congenital pachyonychia. Int J Dermatol 1972; 11: 77-81

28 Wilson AG. Three cases of hereditary hyperkeratosis of the nail-bed. $\mathrm{Br}$ J Dermatol 1905; 17: 13 - 14 